\title{
Trauma Cranioencefálico e Síndrome do Desconforto Respiratório Agudo: Como Ventilar? Avaliação da Prática Clínica*
}

\author{
Traumatic Brain Injury and Acute Respiratory Distress Syndrome: \\ How to Ventilate? Evaluation of Clinical Practice
}

\author{
Lucas Montano Paternostro Saback¹, Mônica Lajana de Almeida², Wandalvo Andrade ${ }^{3}$.
}

\section{RESUMO}

JUSTIFICATIVA E OBJETIVOS: O trauma cranioencefálico (TCE) constitui um problema de saúde mundial, muito destes pacientes evoluem com insuficiência respiratória necessitando de intubação traqueal e suporte ventilatório artificial, apresentando como complicações freqüentes a síndrome do desconforto respiratório agudo (SDRA). Dessa forma, este estudo teve o objetivo de descrever a prática clínica diária sobre o manuseio ventilatório destes pacientes.

MÉTODO: Foram avaliados quais os modos e os parâmetros ventilatórios utilizados para ventilar os pacientes com TCE e SDRA por uma amostra de fisioterapeutas da cidade de Salvador, BA, a partir de um estudo descritivo, por meio de entrevistas face-a-face no período de outubro de 2005 a março de 2006. Para tanto foi elaborado um questionário semi-estruturado contendo

1. Fisioterapeuta, Universidade Católica do Salvador (UCSal) 2. Fisioterapeuta membro da Sobrafir, Fisioterapeuta da UTI do Hospital Geral do Estado da Bahia (HGE), Corpo docente da Faculdade Adventista de Fisioterapia (FAFIS) e da União Metropolitana de Educação e Cultura (UNIME)

3. Fisioterapeuta Coordenador do Hospital Geral do Estado da Bahia (HGE)

*Recebido da Universidade Católica de Salvador (UCSal), Salvador, BA Apresentado à Universidade Católica do Salvador como requisito parcial para a graduação de Bacharel em Fisioterapia.

Apresentado em 29 de agosto de 2006

Aceito para publicação em 19 de fevereiro de 2007

Endereço para correspondência:

Lucas Montano Paternostro Saback

R. Sócrates Guanaes Gomes, 215/704, Cidade Jardim

40296-720 Salvador, BA

Fone: (71) 3452-0458

E-mail: lucasmps@pop.com.br

(C)Associação de Medicina Intensiva Brasileira, 2007 variáveis sócio-demográficas, o perfil do hospital e a estratégia ventilatória aplicada em pacientes com TCE que viessem desenvolver a SDRA.

RESULTADOS: A amostra foi composta por 70 fisioterapeutas, $41(58,6 \%)$ eram do sexo feminino, com média de idade de 31,2 $\pm 6,4$ (24-49) anos e tempo de formado 7,7 $\pm 6,4$ (1-27) anos, dos quais 37 (52,9\%) trabalham em hospital público; 67 (95,7\%) têm alguma especialização. Sessenta e quatro fisioterapeutas afirmam utilizar o modo pressão controlada (PCV). A pressão de pico e a pressão platô desejada para ventilar os pacientes com TCE e SDRA foram em média, de $35,6 \pm 5,3$ (25-50) e 28,4 $\pm 5,8$ (15-35) $\mathrm{cmH}_{2} \mathrm{O}$, respectivamente. Quarenta e oito entrevistados $(68,6 \%)$ afirmaram desejar ventilar os pacientes com TCE e SDRA com a $\mathrm{PaCO}_{2}$ entre 30 e $35 \mathrm{mmHg}$. Trinta e um $(44,3 \%)$ dos entrevistados afirmaram encontrar a PEEP ideal através da PEEP que ofertasse melhor $\mathrm{SpO}_{2} \mathrm{com}$ menor $\mathrm{FiO}_{2}$.

CONCLUSÔES: É incontestável que a estratégia ventilatória de paciente com TCE grave que venha a desenvolver LPA ou SDRA constitua um autêntico desafio; observa-se uma predileção pelo modo PCV devido à já conhecida ventilação com estratégia de proteção pulmonar.

Unitermos: Síndrome do Desconforto Respiratório Agudo, Traumatismo Cranioencefálico, Ventilação Mecânica

\section{SUMMARY}

BACKGROUND AND OBJECTIVES: The traumatic brain injury (TBI) is a healthy-world problem, some of his patients develop respiratory failure, requiring intubation and mechanical ventilation, and the most common complications are the acute respiratory distress syndrome (ARDS). In this way, this study has the ob- 
jective describe the daily clinical practice of respiratory care in this patients submit mechanical ventilation.

METHODS: The methods and ventilatories parameters used to ventilate the patients with TBI and ARDS has been evaluated by a sample of physiotherapists from the city of Salvador, BA, from a descriptive study. The data were collected by face-to-face interviews in the period of October 2005 to March 2006. For in such way a half structuralized questionnaire was elaborated contends changeable social-demographic, about the hospital profile and the applied ventilatory strategy in patients with TBI that come to develop ARDS.

RESULTS: The sample was composed by 70 physiotherapists, 41 (58.6\%) was female, with mean of age of $31.2 \pm 6.4$ (24-49) years-old and graduated time $7.7 \pm 6.4(1-27)$ years, which 37 (52.9\%) works on public hospital; 67 (95.7\%) has any specialization. Sixty four physiotherapists affirm the usage of the pressure controlled ventilation mode. The peak pressure and the plateau pressure wanted to ventilate the patients with TBI and ARDS were in mean 35.6 $\pm 5,3(25-50)$ and $28,4 \pm 5,8(15-35) \mathrm{cmH}_{2} \mathrm{O}$ respectively. Forty eighty $(68.6 \%)$ of the interviewed wants a $\mathrm{PaCO}_{2}$ in $30-35 \mathrm{mmHg}$. Thirty one $(44.3 \%)$ of the interviewed finds the ideal PEEP through the best $\mathrm{SpO}_{2}$ with minor $\mathrm{FiO}_{2}$.

CONCLUSIONS: It's incontestable that the ventilatory strategy of a patient with severe TBI that become to develop ALI or ARDS is an authentic challenge; a predilection for PCV mode is observed due to the already known protective ventilation strategy.

Key Words: Acute Respiratory Distress Syndrome, Mechanical Ventilation, Traumatic Brain Injury

\section{INTRODUÇÃO}

O desenvolvimento de programas de prevenção, a melhoria dos equipamentos de segurança e a legislação de segurança no trabalho tiveram um impacto positivo na epidemiologia das lesões encefálicas ${ }^{1,2}$.

Durante as últimas quatro décadas, o tratamento do traumatismo cranioencefálico (TCE) avançou em muitos aspectos. A implementação de sistemas de rápido transporte e o advento de centros específicos de atendimento, associados aos avanços da Medicina Intensiva e da Neurocirurgia contribuiu para a redução da morbimortalidade destes pacientes ${ }^{1,2}$.

O TCE constitui um problema de saúde mundial, no que diz respeito principalmente à sua etiologia, pois pode ocasionar incapacidade ou óbito, mudar perma- nentemente as habilidades e suas perspectivas e modificar a vida de seus familiares ${ }^{3,4}$.

Correspondem a 200-300 admissões hospitalares por 100 mil habitantes ao ano nos EUA e Inglaterra. Esses números podem variar de acordo com o nível de desenvolvimento do país. Em 2001, na cidade de Salvador, BA, foram atendidas cerca de 11028 vítimas de TCE, no setor de emergência de hospital público de referência, de traumatizados de crânio, destes 555 (5,03\%) necessitaram de internação hospitalar e quanto a letalidade, foram constatados 127 óbitos $^{5}$. Relacionado a isto, têm-se como causas de maior prevalência a violência urbana, os acidentes automobilísticos, as quedas e os acidentes esportivos ${ }^{3,4,6}$. Estima-se que cerca de $30 \%$ a $80 \%$ dos TCE estejam associados ao uso excessivo de álcool etílico ${ }^{7}$.

Os homens jovens, com idade entre 15 e 24 anos, têm maior risco pessoal; as probabilidades de serem vitimados após aos 40 anos diminui para menos de $5 \%$ em ambos os sexos ${ }^{3,4,6}$.

Os pacientes vítimas de TCE podem evoluir com perda do drive respiratório. Nestes casos de TCE grave, em que a escala de Coma de Glasgow varie de 3 a $8^{1,4,8,9}$

A intubação traqueal e a conseqüente dependência da ventilação mecânica tornam-se imprescindível, pois protege as vias aéreas, permite a sedação e a curarização, evita a hipoxemia e previne a hipoventilação, evitando aumentos da pressão intracraniana (PIC) e alterações na pressão de perfusão cerebral (PPC) ${ }^{1}$. Pacientes com lesão no sistema nervoso central (SNC) correm o risco de broncoaspiração, pela diminuição do nível de consciência e perda dos reflexos protetores das vias aéreas ${ }^{9-11}$, podendo causar a síndrome do desconforto respiratório agudo (SDRA), assim como o tempo prolongado na ventilação mecânica ${ }^{1,4}$.

O objetivo deste estudo foi descrever a prática clínica diária sobre o manuseio ventilatório dos pacientes com TCE grave que evoluem com SDRA ao longo de sua permanência na unidade de terapia intensiva (UTI).

\section{MÉTODO}

Foi realizado um estudo descritivo em que se verificou a forma de ventilar os pacientes com TCE e SDRA pelos fisioterapeutas que trabalham em UTI da cidade de Salvador, BA. A coleta de dados foi realizada no período de outubro de 2005 a março de 2006, 
por meio de entrevistas face-a-face, sempre por um mesmo entrevistador, nos hospitais com serviços de emergências gerais e UTI gerais. Diante desses critérios de inclusão foram selecionados cinco hospitais (três privados e dois públicos) como amostra de conveniência para a realização da pesquisa. Foram entrevistados todos os fisioterapeutas que integram a equipe de fisioterapia de cada UTI e os que trabalham em mais de um serviço incluído no estudo, foram entrevistados apenas em uma vez no primeiro local em que foi encontrado.

Previamente à coleta de dados, foi realizado um estudo piloto com cinco fisioterapeutas, de uma das unidades incluídas no estudo, sendo estes não re-abordados para obtenção de dados.

Para a coleta de dados foi utilizado um questionário semi-estruturado contendo variáveis sócio-demográficas, sobre o perfil do hospital e a estratégia ventilatória aplicada em pacientes com TCE que viessem a desenvolver SDRA (Anexo 1). Sobre a estratégia ventilatória foi questionado o modo de ventilação usado (e porquê utiliza este modo), de que maneira é encontrado ou determinado o nível da pressão positiva término expiratória (PEEP), volume-corrente (VT) aplicado, valores desejados para pressão de pico (PPI) e platô (PPL). São ainda variáveis os fatores que implicam em desequilíbrio hemodinâmico, a freqüência e os parâmetros utilizados na terapia de expansão pulmonar (TEP). A análise dos dados foi realizada no programa estatístico de uso livre, SPSS v. 9.0 e o Microsoft Excel 2003. Foi realizada análise descritiva dos dados com o objetivo de verificar as características gerais e específicas da população examinada. No momento da entrevista foi ressaltado que a intenção da pesquisa era descrever a prática clínica diária, não importando os conhecimentos teóricos sobre os itens analisados; e apresentado um termo de consentimento livre e esclarecido, no qual foi exposto os objetivos do estudo para todos os entrevistados, sendo garantido o anonimato, a confidencialidade dos dados e o direito de interromper a entrevista em qualquer momento, de acordo com os aspectos éticos concebidos pela resolução 196/96 do Conselho Nacional de Ética em Pesquisas envolvendo seres humanos.

\section{RESULTADOS}

A amostra foi composta de 81 fisioterapeutas; houve três recusas e um questionário foi anulado por motivos éticos, fisioterapeutas que integrem mais de uma equipe ocorreu em sete situações, sendo entrevistados no primeiro local encontrado. Dos 70 entrevistados, 41 (58,6\%) eram do sexo feminino, com média de idade de 31,2 $\pm 6,4$ (24-49) anos e tempo de formado $7,7 \pm 6,4$ (1-27) anos, dos quais 37 (52,9\%) trabalham em hospital público; 3 (4,3\%) eram graduados, 43 $(61,4 \%)$ tinham alguma especialização em ventilação. Em relação ao perfil dos hospitais incluídos, apenas um hospital particular não era hospital de ensino.

As cinco UTI Gerais totalizaram 116 leitos, destes 62 $(53,5 \%)$ são de hospitais particulares. Foi consenso entre as unidades a monitorização e o registro da ventilação mecânica, e o tempo de permanência da equipe de fisioterapia 24 horas por dia. Vinte e cinco dos entrevistados $(35,7 \%)$ afirmaram haver monitorização de PIC em todos pacientes com TCE e SDRA, destes, $23(32,8 \%)$ trabalham em hospital particular; e 27 (38,6\%) afirmaram haver monitorização da capnometria em todos pacientes com TCE e SDRA, destes, $22(31,6 \%)$ trabalham em hospital particular. Em relação aos ventiladores disponíveis em cada UTI e qual o de melhor nível técnico para ventilar os pacientes com TCE e SDRA, 55 (78,57\%), afirmaram estar usando o considerado de melhor nível técnico para ventilar os pacientes com TCE e SDRA, de acordo com a tabela 1. Sessenta e quatro fisioterapeutas $(91,4 \%)$ afirmaram utilizar o modo pressão controlada (PCV) para ventilar os pacientes com TCE e SDRA; destes $55,7 \%$ (39) utilizam com o objetivo de proteger as vias aéreas, $18,6 \%$ (13) para melhor monitorizar as pressões e 5,7\% (4) afirmaram seguir protocolo da unidade, apesar de não haver protocolo em nenhuma unidade, em posterior investigação. Houve perda de dados, $n=8(11,4 \%)$, de acordo com a figura 1 .

Tabela 1 - Modelos de Respiradores Disponíveis em cada UTI

\begin{tabular}{lc}
\hline Respiradores & Em quantas UTI? \\
\hline Evita 1 & 1 \\
Evita 2 & 1 \\
Evita 2 Dura & 2 \\
Evita 4 Dura & 2 \\
New Port & 2 \\
Takaoka & 1 \\
Inter 5 & 2 \\
Inter Plus & 2 \\
Bird 6400 & 1 \\
Savina & 1 \\
Servo $900 \mathrm{C}$ & 1 \\
Bear 1000 & 1 \\
Vela & 2 \\
\hline
\end{tabular}




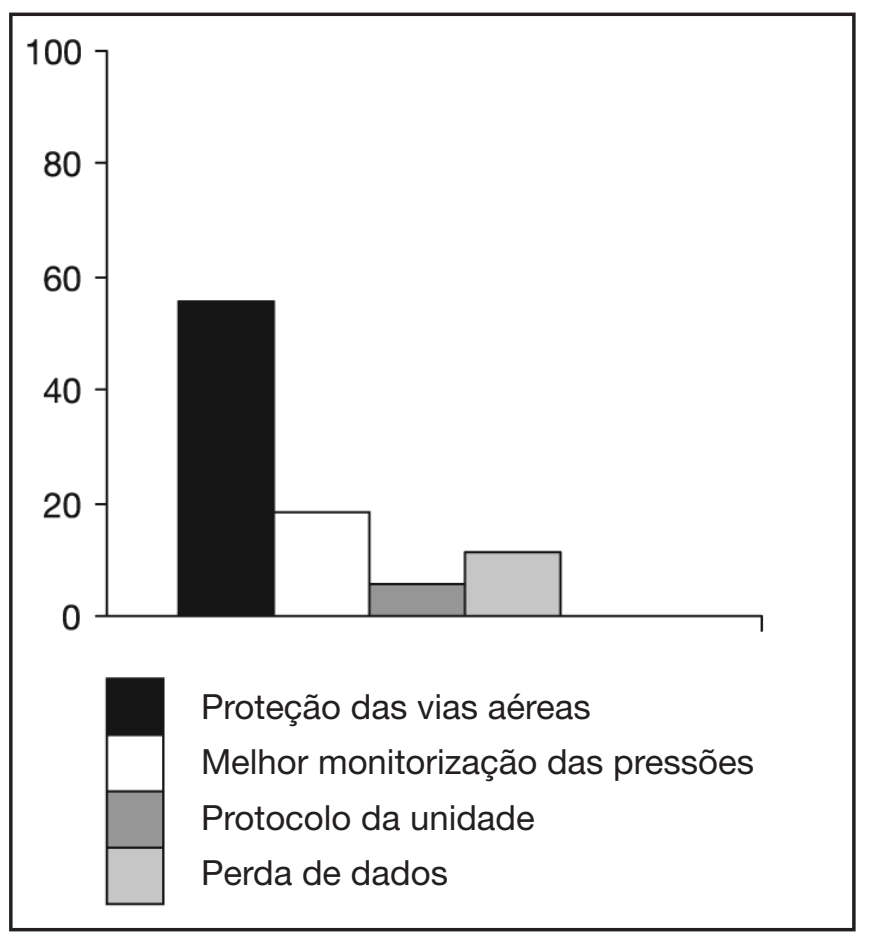

Figura 1 - Indicação, em Freqüência Relativa, da Utilização do Modo PCV em Pacientes com TCE e SDRA.

Seis entrevistados $(8,6 \%)$ afirmaram utilizar o modo volume controlado (VCV) para ventilar os pacientes com TCE e SDRA, destes 2,9\% (2) utilizam como o objetivo de garantir o VT ideal para o paciente, e 2,9\% (2) seguiam protocolo da unidade, apesar de não haver protocolo em nenhuma unidade, em posterior investigação. Houve perda de dados, $n=2$ (2,9\%). A PPI desejada para ventilar os pacientes com TCE e SDRA foi em média de $35,6 \pm 5,3(25-50) \mathrm{cmH}_{2} \mathrm{O}$, já a PPL desejada para ventilar os pacientes com TCE e SDRA foi de $28,4 \pm 5,8$ (15-35) $\mathrm{cmH}_{2} \mathrm{O}$. Quarenta e oito entrevistados $(68,6 \%)$ afirmaram desejar ventilar os pacientes com TCE e SDRA numa $\mathrm{PaCO}_{2}$ entre 30 e $35 \mathrm{mmHg}$ (Figura 2). Trinta e um (44,3\%) dos entrevistados afirmaram encontrar a PEEP ideal para ventilar os pacientes com TCE e SDRA através da PEEP que oferte a melhor $\mathrm{SpO}_{2}$ com menor $\mathrm{FiO}_{2}$ (Figura 3).

Sessenta e sete $(95,7 \%)$ fisioterapeutas afirmaram realizar TEP no pacientes com TCE e SDRA; destes, 56 $(80 \%)$ utilizam a PEEP, nas suas diversas formas de aplicação, como técnica de escolha. A PEEP média para o recrutamento foi de $30,6 \pm 5,6(20-40) \mathrm{cmH}_{2} \mathrm{O}$, e como critério para realização da técnica, $57,1 \%$ ( $n=$ 40) dos entrevistados utilizam indicadores funcionais, como radiografias, hemogasometria, ausculta pulmonar e $\mathrm{SpO}_{2}$ (Figura 4).

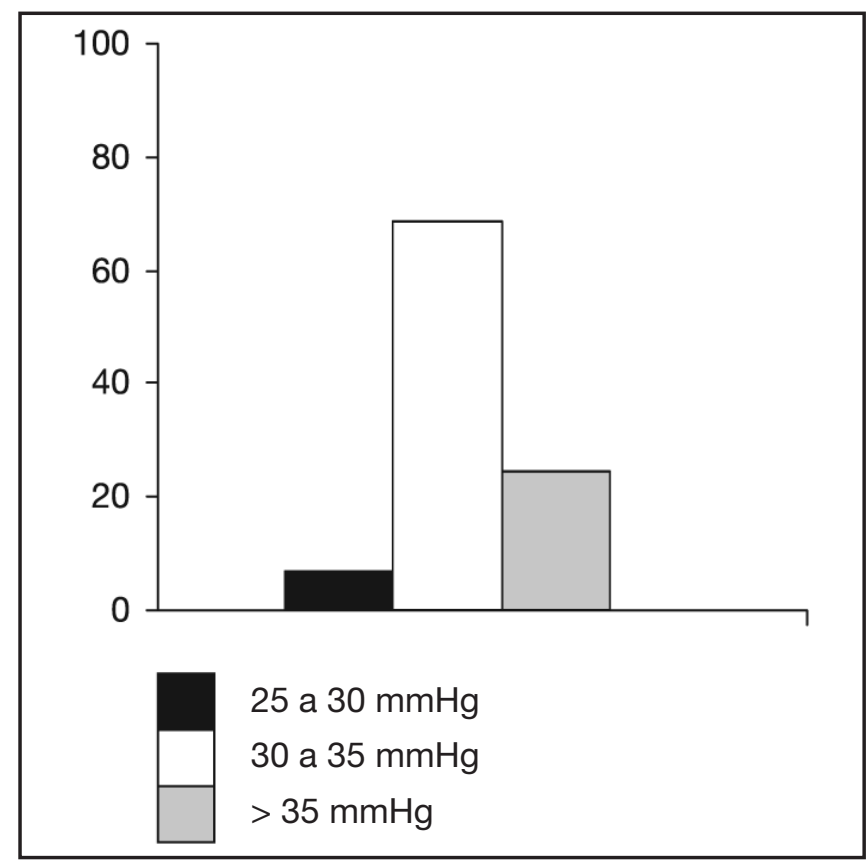

Figura 2 - Determinação, em Freqüência Relativa, da Forma Relatada pelos Fisioterapeutas, de como é Encontrada a $\mathrm{PaCO}_{2}$ Desejada para Ventilar os Pacientes com TCE e SDRA $(n=70)$

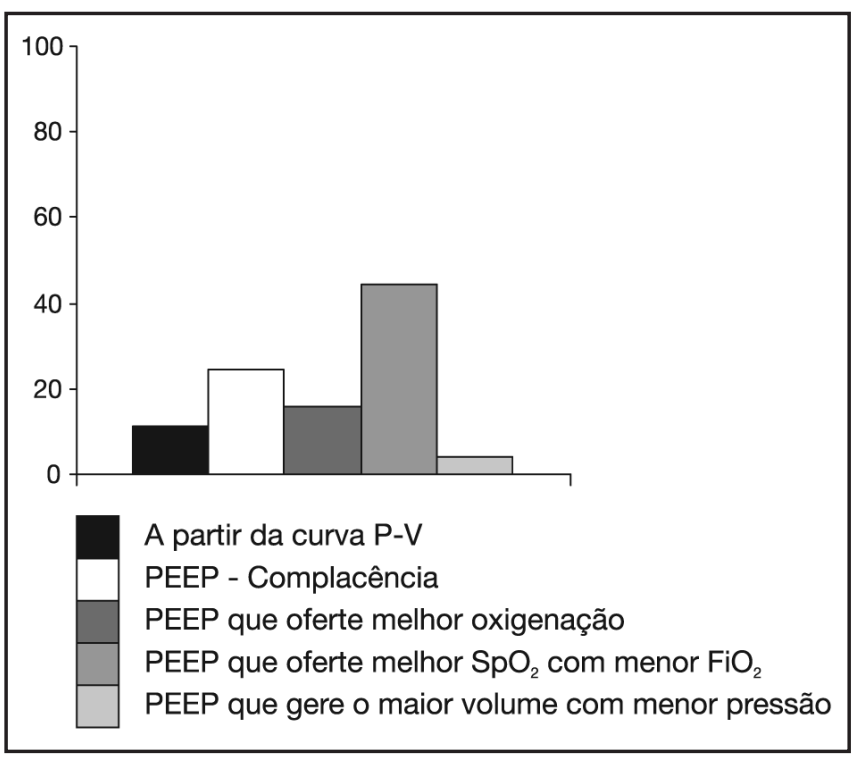

Figura 3 - Determinação, em Freqüência Relativa, da Forma Relatada pelos Fisioterapeutas, de como é Encontrada a PEEP Ideal para Ventilar os Pacientes com TCE e SDRA $(n=70)$ 


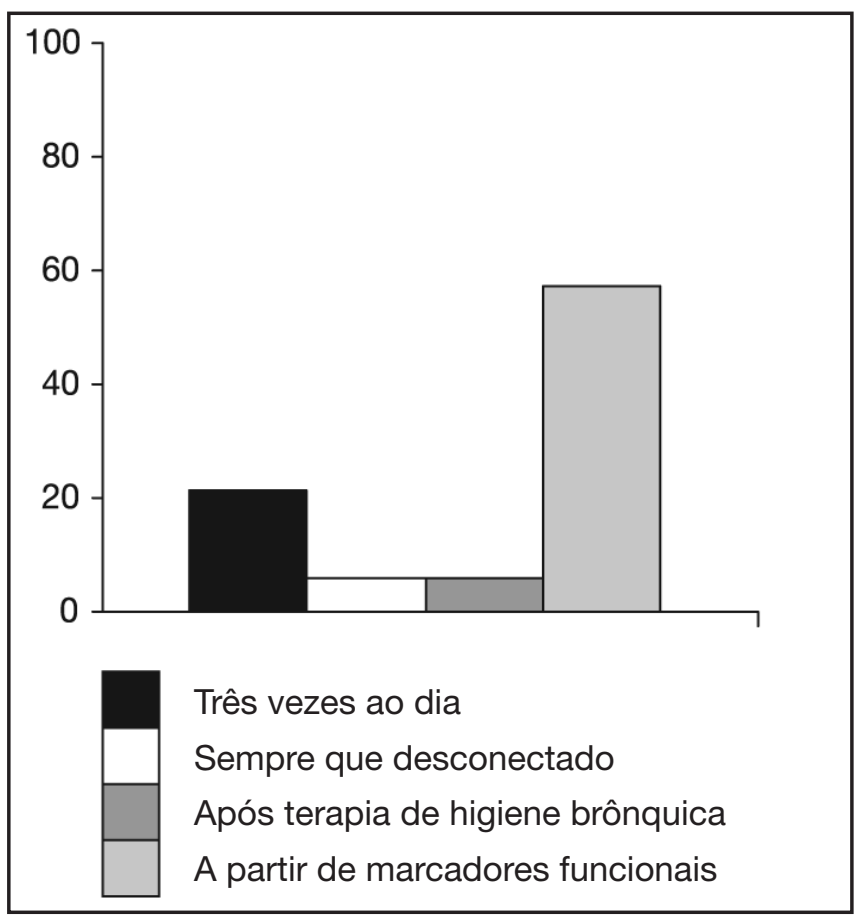

Figura 4 - Determinação, em Freqüência Relativa, dos Critérios Relatados pelos Fisioterapeutas, para a Realização da Terapia de Expansão Pulmonar nos Pacientes com TCE e SDRA $(n=67)$

Caso não houvesse monitorização da PIC, apenas $37(52,9 \%)$ fisioterapeutas realizariam a TEP, desses, $31,5 \%$ utilizariam a PEEP, com valor médio de 31,2 $\pm 5,2$ (20-35) $\mathrm{cmH}_{2} \mathrm{O}$, o restante, $21,4 \%$ realizariam o suspiro com TEP.

\section{DISCUSSÃO}

Foi observado que a modalidade encontrada para ventilar os pacientes com TCE e SDRA é o modo pressão controlada (PCV); encontram a PEEP ideal através da maior $\mathrm{SpO}_{2}$ com menor $\mathrm{FiO}_{2}$; utilizam hiperventilação leve $\left(\mathrm{PaCO}_{2}\right.$ entre 30 e $\left.35 \mathrm{mmHg}\right)$; $\mathrm{PPI}$ e PPL com valores de $35,6 \pm 5,3$ e $28,4 \pm 5,8$ $\mathrm{cmH}_{2} \mathrm{O}$, respectivamente; e uma PEEP média para o recrutamento alveolar desses pacientes $30,6 \pm 5,6$ $\mathrm{cmH}_{2} \mathrm{O}$.

As estratégias terapêuticas em pacientes com lesão encefálica nas UTI têm se voltado para o tratamento e a prevenção da isquemia encefálica como também da lesão pulmonar aguda e síndrome do desconforto respiratório agudo ${ }^{12}$. O manuseio da hipertensão intracraniana e o cuidado em se evitar a hipotensão sistêmica constituem o suporte prin- cipal no cuidado do paciente crítico com traumatismo cranioencefálico grave ${ }^{13}$. Torna-se imprescindível a atenção e os cuidados básicos ao paciente com TCE que venham a alterar a hemodinâmica e conseqüentemente o aumento da hipertensão intracraniana. Fatores como elevação demasiada da cabeceira (acima de $30^{\circ}$ ), aspiração endotraqueal (com aumento da $\mathrm{PaCO}_{2}$, resultando em vasodilatação encefálica), tosse, febre e o menor esforço do paciente pode estar associado com a elevação da PIC 8,913-19. Apesar da maioria dos entrevistados $(64,3 \%)$ afirmarem não haver monitorização da PIC, Cremer e col. ${ }^{20}$ observaram que apenas $6 \%$ dos pacientes se beneficiariam de uma terapia-alvo para PIC (<20 mmHg) e PPC (> $70 \mathrm{mmHg})$, considerando um risco absoluto de $90 \%$. Em outras palavras, para que um paciente se beneficiasse, precisaria de um número superior ou igual a 16 sendo trata$\mathrm{do}^{20}$. A vasoconstrição induzida pela hipocapnia e a redução subseqüente no fluxo sanguíneo cerebral (FSC) podem diminuir rapidamente a PIC. Podem ser usados breves períodos de hiperventilação leve $\left(\mathrm{PaCO}_{2}\right.$ entre 30 e $\left.35 \mathrm{mmHg}\right)$ como parte do manuseio da PIC ${ }^{2}$. Observou-se concordância entre os dados da literatura e os resultados desta pesquisa, onde $68,7 \%$ desejam a $\mathrm{PaCO}_{2}$ entre 30 e $35 \mathrm{mmHg}{ }^{4}$ para ventilar os pacientes com TCE e SDRA. A hiperventilação prolongada deve ser evitada na ausência de PIC elevada, e a hiperventilação profilática deve ser evitada nas primeiras $24 \mathrm{~h}$ de lesão encefálica, porque neste período é comprovado que o FSC é reduzido a menos da metade ${ }^{1,2,4}$. Entre as complicações mais freqüentes e graves associadas ao paciente com TCE grave, destacam-se a LPA e SDRA, que se beneficiariam da aplicação da PEEP e da ventilação com proteção pulmonar ${ }^{1}$. Observou-se na literatura incidência entre $20 \%$ e $81 \%$ do comprometimento pulmonar nos pacientes com lesão encefálica ${ }^{1,16,21,22}$. A aplicação da PEEP nos pacientes criticamente enfermos, após TCE, permanece controverso. Clinicamente, a PEEP exerce efeitos benéficos na oxigenação pelo aumento da capacidade residual funcional, redução do shunt intrapulmonar, pelo recrutamento alveolar e redução da suplementação de oxigênio. PEEP em excesso, por outro lado, pode causar volutrauma, dificultar a drenagem venosa, reduzir a PAM e aumentar a PIC 13. No presente estudo, 44,3\% da amostra afirmou encontrar a PEEP basal dos pacientes com TCE e SDRA através da PEEP que oferte melhor $\mathrm{SpO}_{2} \mathrm{com}$ 
menor $\mathrm{FiO}_{2}$; e a PEEP para a manobra de recrutamento nesses pacientes $30,6 \pm 5,6 \mathrm{cmH}_{2} \mathrm{O}$. Em concordância, Frost e col. não observaram alterações na PIC com o aumento da PEEP em $40 \mathrm{cmH}_{2} \mathrm{O}$ 23. Já Huynh e col. demonstraram que a aplicação da PEEP de $20 \mathrm{cmH}_{2} \mathrm{O}$ não ocasionou elevação da PIC ou produziram mudanças significativas na PPC, mesmo em pacientes com complacência intracraniana reduzida e hipertensão intracraniana ${ }^{13}$. Num estudo envolvendo 33 pacientes com lesão encefálica grave submetida a PEEP de no máximo 15 $\mathrm{cmH}_{2} \mathrm{O}$, resultou em aumento clinicamente insignificante da PIC, de 1,3 mmHg. Destes 33 pacientes, observou-se elevação da PIC em 17, diminuição em cinco, e permaneceu inalterada de acordo com outros estudos ${ }^{11,14}$. Georgiadis e col. ${ }^{18}$ mostraram que a aplicação da PEEP de $12 \mathrm{mmHg}$ não apresentou influência significativa na PIC em pacientes com doença céfalo-vascular, e a diminuição na PPC foram devido a mudanças na PAM. Segundo Caricato e col. ${ }^{15}$ o aumento da PEEP de 0 a $12 \mathrm{cmH}_{2} \mathrm{O}$ não apresentou efeitos significativos na PIC em pacientes com TCE, com ou sem alterações pulmonares. Por outro lado, a aplicação da PEEP com valores acima ou iguais a $15 \mathrm{cmH}_{2} \mathrm{O}$ pode agravar ou induzir a hipertensão intracraniana ${ }^{12}$. Shapiro e col. fizeram a aplicação da PEEP, de causa traumática ou não e LPA (dentre os quais dois pacientes apresentavam relação $\mathrm{PaO}_{2} / \mathrm{FiO}_{2}<200 \mathrm{mmHg}$ ), com o propósito de investigar os efeitos das manobras de recrutamento a volume de acordo com a função respiratória, em 12 pacientes com lesão encefálica a PIC elevou-se em $10 \mathrm{mmHg}$ ou mais em seis dos 12 pacientes em que foram aplicados PEEP de 4 a $8 \mathrm{cmH}_{2} \mathrm{O}$, resultando em diminuição da PPC abaixo de $50 \mathrm{mmHg}$. Bein e col. ${ }^{12}$ ventilaram 11 pacientes com lesão encefálica aguda (MRV) sobre a PIC e o metabolismo encefálico. Durante a MRV (elevação da driving pressure para $60 \mathrm{cmH}_{2} \mathrm{O}$ ) observou-se significativa diminuição da PAM e elevação da PIC, resultando em diminuição da PPC para valores abaixo de 65 $\mathrm{mmHg}{ }^{5,12}$. No presente estudo os valores da mecânica respiratória, "PPI e PPL", encontrados foram de $35,6 \pm 5,3$ e $28,4 \pm 5,8 \mathrm{cmH}_{2} \mathrm{O}$, respectivamente; não há dados objetivos na literatura que possam discutir essa variável, no entanto esses valores estão dentro dos limites aplicáveis em pacientes com SDRA isoladamente ${ }^{24,26,28,31}$. Além disso, vêm sendo observado que a mecânica respiratória nos pacientes com lesão encefálica e pneumonia associada à ventilação, apresenta aumento da pressão intra-abdominal, da elastância da parede torácica e da resistência das vias aéreas ${ }^{22}$. Em pacientes com baixa complacência do sistema respiratório e lesão encefálica, a aplicação da PEEP (12 $\left.\mathrm{cmH}_{2} \mathrm{O}\right)$ não apresentou efeito significativo na hemodinâmica encefálica e sistêmica ${ }^{15}$. Alguns autores têm acreditado que fatores que reduzam a complacência pulmonar, como pneumonia ou atelectasia, tenham efeito de proteção para diminuir a transmissão da pressão das vias aéreas ${ }^{15}$. Em relação ao modo ventilatório utilizado $91,4 \%$ dos entrevistados afirmaram utilizar o modo pressão controlada (PCV). Não há dados na literatura favorecendo um modo ou outro; questão de extrema relevância, citado por Georgiadis e col. ${ }^{18}$ "nenhum estudo tem avaliado o modo ventilatório ideal para os pacientes com doença encéfalo-vascular, particularmente para os pacientes com PIC elevada que desenvolvem pneumonia ou SDRA". Supõe-se que a predileção pelo modo PCV deva-se pela estratégia de ventilação de proteção pulmonar, onde se preconiza a utilização de baixo volume-corrente e pressão limitada nas vias aéreas para evitar a hiperdistensão alveolar, juntamente com alto ou moderado valor da PEEP, para prevenir os colapsos alveolares, resultando em melhor sobrevida dos pacientes com SDRA ${ }^{25-31}$.

\section{CONCLUSÃO}

Observou-se neste estudo uma grande discrepância entre os dados obtidos em uma mesma UTI, possivelmente pela ausência de protocolo ou fatores individuais profissionais. Outro fator que deve ser citado foi a considerável perda de dados, mesmo sendo enfatizado que não estaria sendo avaliado os conhecimentos teóricos em relação ao assunto proposto. Uma possível causa seja pelo antagonismo às condutas dos pacientes com TCE e SDRA separadamente.

É incontestável que a estratégia ventilatória de um paciente com TCE grave que venha a desenvolver LPA ou SDRA constitua um autêntico desafio. As medidas terapêuticas específicas do TCE se unem à necessidade de ventilação de proteção pulmonar, resultando, muitas vezes, em manobras terapêuticas antagônicas. Mais estudos aleatório e controlado precisam ser realizados para se determinar técnicas alternativas e qual o modo ideal de ventilar estes pacientes. 
Anexo 1 - TCE x SDRA: Como ventilar?

Questionário de Pesquisa

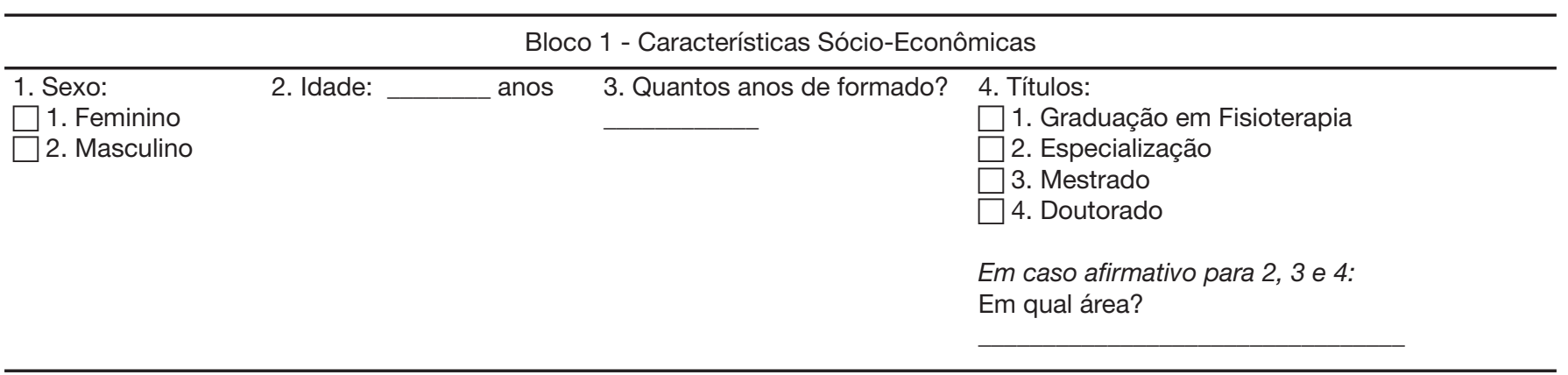

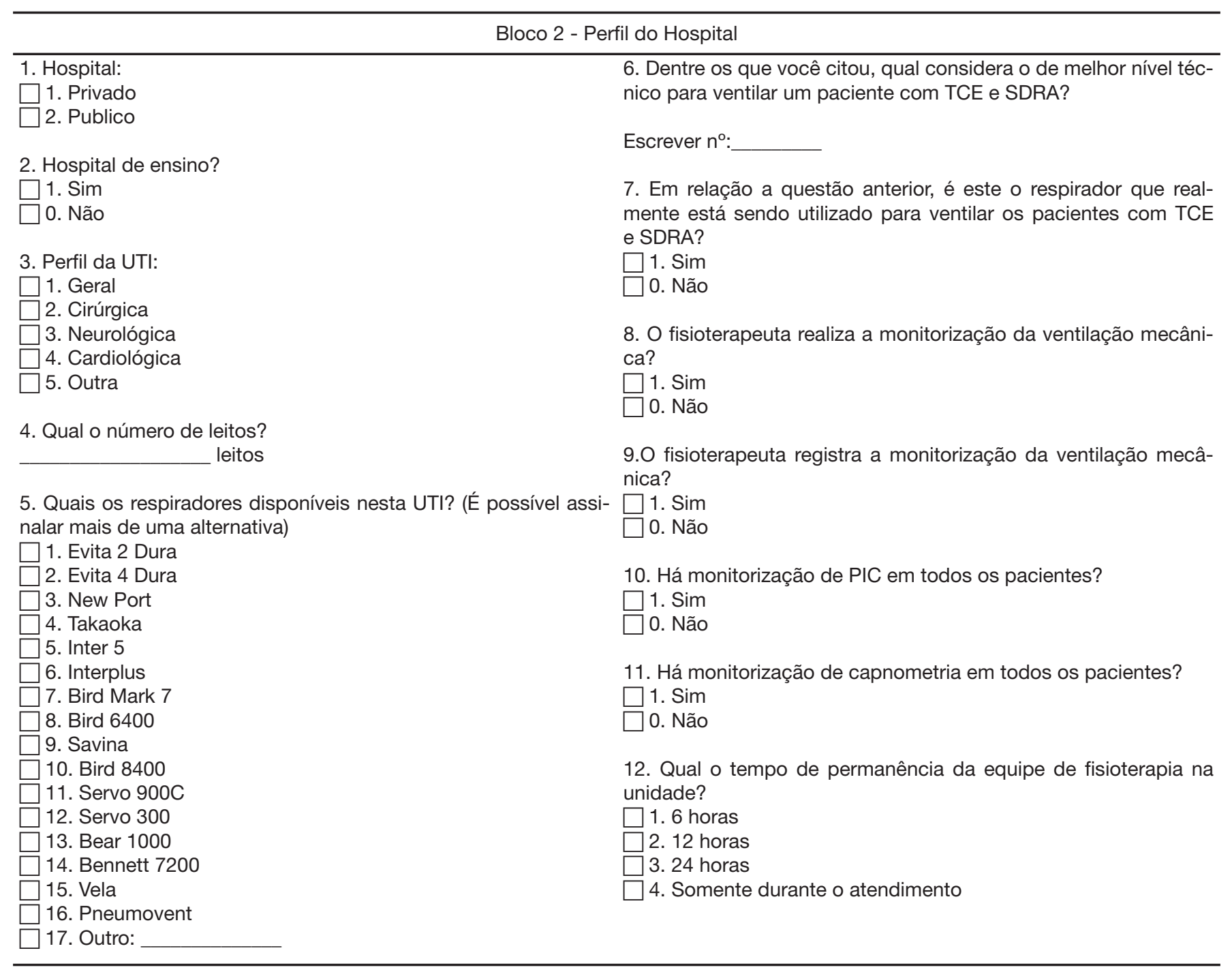




\section{Bloco 3 - Estratégia Ventilatória}

1. Qual o modo ventilatório usado nos pacientes com TCE e 8. Na sua opinião, qual o valor de pressão platô $\left(P_{p}\right)$ SDRA?

$\square$ 1. VCV (Ventilação controlada a volume)

2. PCV (Ventilação controlada a pressão)

2. Se a resposta da questão 1 for 1 . Quais os parâmetros utilizados? Se a resposta foi 2, pule para questão 3 .

FR:

VT: $\mathrm{mL} / \mathrm{kg}$ desejado para ventilar os pacientes com TCE e SDRA?

I:E:

Fluxo:

3. Se a resposta da questão 1 for 2. Quais os parâmetros utilizados?

Pressão Contr.: $\mathrm{mL} / \mathrm{kg} \quad$ T. insp.:

FR: $\mathrm{I}: \mathrm{E}:$

4. Porque você utiliza este modo ventilatório?

5. Em relação a monitorização das trocas gasosas pela gasometria, qual $\mathrm{O}$ valor desejado de $\mathrm{PaCO}_{2}$ para ventilar os pacientes com TCE e SDRA?
1. $<25 \mathrm{mmHg}$
2. 25 a $30 \mathrm{mmHg}$
3. 30 a $35 \mathrm{mmHg}$
4. $>35 \mathrm{mmHg}$

6. De que maneira é encontrado (ou determinado) o nível da PEEP adequado para ventilar os pacientes com TCE e SDRA?
$\square$ 1. A partir da curva $P \times V$
2. PEEP - Complacência
3. PEEP que oferte a melhor oxigenação
4. PEEP que oferte a melhor $\mathrm{SpO}_{2}$ com menor $\mathrm{FiO}_{2}$
5. PEEP que gere o $>$ volume com $<$ pressão

7. Na sua opinião, qual o valor de pressão de pico $\left(P_{\mathrm{p}}\right)$ desejado para ventilar os pacientes com TCE e SDRA?
9. Nos pacientes em ventilação mecânica com TCE e SDRA, você faz terapia de expansão pulmonar?

$\square$ 1. Sim

0. Não

10. Qual a técnica utilizada?

11. Quais os parâmetros que são utilizados para a terapia de expansão pulmonar?

12. Com que freqüência você realiza a terapia de expansão pulmonar?
$\square 1.1$ vez ao dia
2. 2 vezes ao dia
3. 3 vezes ao dia
4. Sempre que desconectado

5. Após a terapia de higiene brônquica

6. Outro:

13. Caso não haja a monitorização da pressão intracraniana (PIC), você realizaria a terapia de expansão pulmonar?

$\square$ 1. Sim

0. Não

14. Qual a técnica utilizada?

15. Quais os parâmetros que são utilizados para a terapia de expansão pulmonar? 


\section{REFERÊNCIAS}

01. Belda FJ, Aguilar, Soro M et al - Ventilatory management of the severely brain-injured patient. Rev Esp Anestesiol, 2004;51:143-150.

02. Vender JR - Hyperventilation in severe brain injury revisited. Crit Care Med, 2000;28:3361-3362

03. Stokes M - Neurologia para Fisioterapeutas; $1^{\mathrm{a}}$ Ed, São Paulo, Premier, 2000;101-115.

04. Dutton RP, McCunn M - Traumatic brain injury. Curr Opin Crit Care 2003;9:503-509.

05. Melo JRT, Silva RA, Moreira ED - Características dos pacientes com trauma cranioencefálico na cidade do Salvador, Bahia, Brasil. Arq Neuropsquiatr, 2004;62:711-715.

06. Pereira CU - Neurogeriatria. $1^{\text {a }}$ Ed, Rio de Janeiro: Revinter, 2001;344345.

07. Knobel E - Terapia Intensiva - Neurologia. $1^{\text {a }}$ Ed, São Paulo: Atheneu, 2003

08. Dantas VP, Falcão ALE, Sardinha LAC et al - Fatores que influenciaram a evolução de 206 pacientes com traumatismo cranioencefálico grave. Arq Neuropsquiatr, 2004;62(2-A):313-318

09. Thiesen RA, Dragosavac D, Roquejani AC et al - Influência da fisioterapia respiratória na pressão intracraniana em pacientes com traumatismo cranioencefálico grave. Arq Neuropsquiatr, 2005;63:110-113.

10. André C, Freitas GR - Terapia Intensiva em Neurologia e Neurocirurgia; $1^{\text {a }}$ Ed, Rio de Janeiro: Revinter, 2002.

11. Davis DP, Idris AH, Sise MJ et al - Early ventilation and outcome in patients with moderate to severe traumatic brain injury. Crit Care Med, 2006;34:1202-1208

12. Bein T, Kuhr LP, Bele S et al - Lung recruitment maneuver in patients with cerebral injury: effects on intracranial pressure and cerebral metabolism. Intensive Care Med, 2002;28:554-558.

13. Huynh T, Messer M, Sing RF et al - Positive end-expiratory pressure alters intracranial and cerebral perfusion pressure in severe traumatic brain injury. J Trauma, 2002;53:488-493.

14. Cooper KR, Boswell PA, Choi SC - Safe use of PEEP in patients with severe head injury. J Neurosurg, 1985;63:552-555

15. Caricato A, Conti G, Della Corte F et al - Effects of PEEP on the intracranial system of patients with head injury and subarachnoid hemorrhage: the role of respiratory system compliance. J Trauma, 2005;58:571-576.

16. Mascia L, Grasso S, Fiore T et al - Cerebro-pulmonary interactions during the application of low levels of positive end-expiratory pressure. In- tensive Care Med, 2005;31:373-379

17. Melo JRT, Oliveira J, Silva RA, Moreira ED et al - Fatores preditivos do prognóstico em vítimas de trauma cranioencefálico. Arq Neuropsquiatr, 2005;63:1054-1057

18. Georgiadis D, Schwarz S, Baumgartner RW et al - Influence of positive end-expiratory pressure on intracranial pressure and cerebral perfusion pressure in patients with acute stroke. Stroke, 2001;32:2088-2092.

19. Schwarz S, Georgiadis D, Aschoff A - Effects of body position on intracranial pressure and cerebral perfusion in patients with large hemispheric stroke. Stroke, 2002;33:497-501.

20. Cremer OL, Van Dijk GW, van Wensen E et al - Effect of intracranial pressure monitoring and targeted intensive care on functional outcome after severe head injury. Crit Care Med, 2005;33:2207-2213.

21. Martinez-Perez M, Bernabe F, Pena R et al - Effects of expiratory tracheal gas insufflation in patients with severe head trauma and acute lung injury. Intensive Care Med, 2004;30:2021-2027.

22. Gamberoni C, Colombo G, Aspesi M et al - Respiratory mechanics in brain injured patients. Minerva Anestesiol, 2002;68:291-296.

23. Frost $\mathrm{E}$ - Effects of positive end-expiratory pressure on intracranial pressure and compliance in brain-injured patients. J Neurosurg, 1977;47:195-200.

24. Shapiro HM, Marshall LF - Intracranial pressure responses to PEEP in head-injured patients. J Trauma, 1978;18:254-256.

25. Villagra A, Ochagavia A, Vatua $S$ et al - Recruitment maneuvers during lung protective ventilation in acute respiratory distress syndrome. Am J Respir Crit Care Med, 2002;165:165-170.

26. Eichacker PQ, Gerstenberger EP, Banks SM et al - Meta-analysis of acute lung injury and acute respiratory distress syndrome trials testing low tidal volumes. Am J Respir Crit Care Med, 2002;166:1510-1514.

27. Pinsky MR - Toward a better ventilation strategy for patients with acute lung injury. Crit Care, 2000;4:205-206.

28. Brower RG, Ware LB, Berthiaume $Y$ et al - Treatment of ARDS. Chest, 2001;120:1347-1367.

29. Kaplan LJ, Bailey H, Formosa V - Airway pressure release ventilation increases cardiac performance in patients with acute lung injury/adult respiratory distress syndrome. Crit Care, 2001;5:221-226.

30. Bellingan GJ - The pulmonary physician in critical care: The pathogenesis of ALI/ARDS. Thorax, 2002;57:540-546.

31. Amato MB, Barbas CS, Medeiros DM et al - Effect of a protective-ventilation strategy on mortality in the acute respiratory distress syndrome. $\mathrm{N}$ Engl J Med, 1998;338:347-354. 